\title{
Charged-Particle Cross Section Database for Medical Radioisotope Production: Chapter 3, Theoretical Evaluations
}

\author{
Mohammed G. Mustafa
}

June 24, 1999

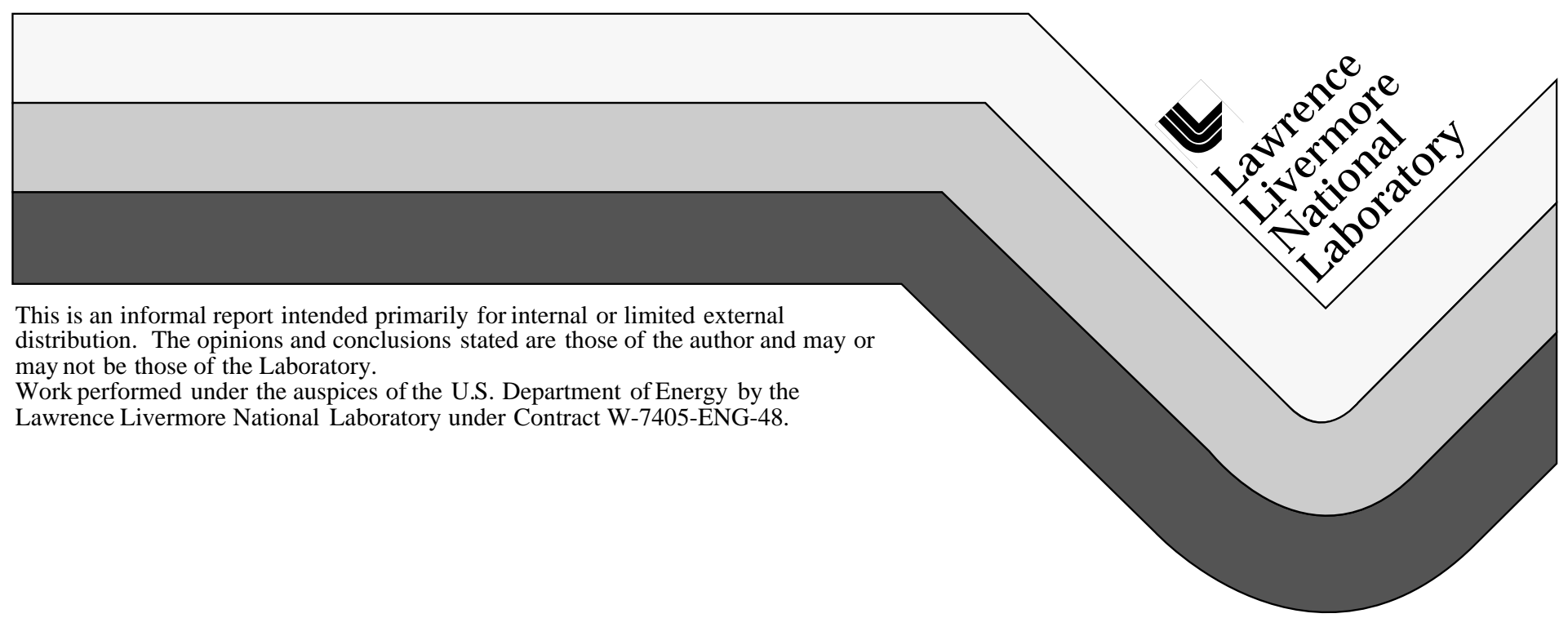




\section{DISCLAIMER}

This document was prepared as an account of work sponsored by an agency of the United States Government. Neither the United States Government nor the University of California nor any of their employees, makes any warranty, express or implied, or assumes any legal liability or responsibility for the accuracy, completeness, or usefulness of any information, apparatus, product, or process disclosed, or represents that its use would not infringe privately owned rights. Reference herein to any specific commercial product, process, or service by trade name, trademark, manufacturer, or otherwise, does not necessarily constitute or imply its endorsement, recommendation, or favoring by the United States Government or the University of California. The views and opinions of authors expressed herein do not necessarily state or reflect those of the United States Government or the University of California, and shall not be used for advertising or product endorsement purposes.

This report has been reproduced directly from the best available copy.

Available to DOE and DOE contractors from the Office of Scientific and Technical Information P.O. Box 62, Oak Ridge, TN 37831

Prices available from (423) 576-8401

Available to the public from the National Technical Information Service

U.S. Department of Commerce 5285 Port Royal Rd. Springfield, VA 22161 


\section{Chapter 3. Theoretical Evaluations}

Creation of a Reference Charged Particle Cross Section Database for Medical Radioisotope Production requires the evaluation of both experimental and modeled cross sections for beam monitor reactions and for radionuclide (positron and gamma emitters) production reactions. It was recognized at the first meeting of this CRP in Vienna in 1995 that modeling will play an important role in predicting cross sections where measurements are either not available or have large discrepancies. Because of the volume of work involving about forty-five reactions in the CRP, it was decided to use modeling as a guide rather than for full evaluation. (Although in some cases the CRP used the modeled cross sections as the recommended values). Thus the modeling was done using global input parameters. In this chapter we describe the modeling by four different groups: Livermore, Obninsk, Beijing and Islamabad. First we give a general overview of nuclear reaction models that may be used in modeling cross sections below $100 \mathrm{MeV}$. This will be followed by a short description of the codes and calculations actually used by the four groups. (We note that the codes have similar basic reaction physics, but they differ in details and in actual applications.) In the final section we give a discussion of the modeling with its successes and failures in reproducing experimental data using global input parameters.

\subsection{Nuclear reaction models}

The specific reactions involved in this CRP are given in earlier sections. The energies for these reactions range from the threshold (several MeV) to about one hundred $\mathrm{MeV}$ with protons, deuterons, ${ }^{3} \mathrm{He}$ and alpha particles as projectiles, and targets ranging from light (nitrogen) to heavy (bismuth) masses. The nuclear reaction theories and models covering the target-projectile and energy ranges relevant to this CRP include various preequilibrium models (Blann, 1975; Gadioli and Hodgson, 1992) coupled with the Hauser-Feshbach theory (1952) or the Weisskopf-Ewing evaporation model (1940). Intranuclear cascade models (Bertini, 1963; Chen et al., 1968; Gudima et al., 1983; Cugnon et al., 1997) could be used in this energy regime, but have not been used for this CRP. The quantum mechanical, multistep direct reaction theories (Feshbach et al., 1980; Udagawa et al., 1982; Nishioka et al., 1988) have started to play a role in this energy range, but the modeling has not yet matured to the level of a routine application to data 
evaluations. Also R-matrix calculations (Lane and Thomas, 1958), which are quite complex but more appropriate for lighter targets, such as, ${ }^{14,15} \mathrm{~N}$ and ${ }^{16,18} \mathrm{O}$, are not done here. The CRP relied on evaluation of the experimental cross sections to obtain the recommended values for targets below $\mathrm{A}<30$. For other targets the cross sections were modeled using preequilibrium-evaporation formalisms, as described below and in the following section.

The commonly used preequilibrium models are the exciton model and the hybrid model (Gadioli and Hodgson, 1992; Blann, 1975). These semiclassical models both originate from the pioneering paper, "Statistical Model of Intermediate Structure", by J. J. Griffin (1966). The nuclear state is characterized by the excitation energy of the composite nucleus and the exciton number, which is the total number of particles above and holes below the Fermi surface. It is assumed that all possible ways of sharing the excitation energy between different particle-hole configurations with the same exciton number have equal a-priori probability. The exciton number changes during the reaction process as a result of intranuclear two-body collisions. At each stage of the reaction there may be a non-zero probability that a particle is emitted. If this happens at an early stage, we speak of pre-equilibrium emission. If the emission does not occur at an early stage, the system eventually reaches the equilibrium or evaporation stage. This stage is described by the Weisskopf-Ewing formalism (1940) (which does not treat angular momentum and parity explicitly) or more rigorously by the Hauser-Feshbach formalism (1952) which explicitly treats vector coupling of spins and parities between compound and residual nuclei and ejectiles. Preequilibrium models have been widely used in modeling nuclear cross sections below $200 \mathrm{MeV}$ and have provided an adequate description of the high-energy tails (i. e., the region between the evaporation peak and the discrete states) of the outgoing particle spectra (Blann et al., 1994; Michel and Nagel, 1997). The details of the preequilibrium and evaporation models are given as appropriate in the next section on "codes and calculations". Here the essential ideas are provided with some simple expressions.

Several formulations of PE decay are in use; these are the 'hybrid', the 'geometry dependent hybrid' (GDH) and the 'exciton' model formulations. These approaches rely on a quantity called the "partial state density," which is the number (per MeV) of energy partitions available for a Fermi gas where every partition of $\mathrm{p}$ particles and h holes is 
assumed to occur with equal a-priori probability. The first expression for this partial state density was due to Ericson (1960),

$$
\rho_{n}(E)=g(g E)^{n-1} /(p ! h !(n-1) !)
$$

where $n$, the exciton number, equals the number of excited particles " $p$ " plus holes " $h$," $E$ is the excitation energy in $\mathrm{MeV}$, and $g$ is the single particle level density at the Fermi energy. PE decay models in use make the assumption that within each exciton hierarchy, all configurations are populated with equal a-priori probability.

The second quantity in the Griffin ('exciton') model is the exciton-exciton transition rate. This may be given by the "golden rule" of the first-order time-dependent perturbation theory,

$$
\lambda_{n n^{\prime}}=\frac{2 \pi}{\hbar}|M|^{2} \rho_{n^{\prime}}(E)
$$

where $|M|^{2}$ is the square of the matrix element corresponding to a residual two-body interaction. For most applications an energy- and mass-dependent average value for $|M|^{2}$ is used.

The probability for the preequilibrium emission of a particle with energy $\varepsilon$ is given by

$$
W_{p}(\varepsilon) d \varepsilon=\sum_{n} \frac{\rho_{n-1}(U) \rho_{c}(\varepsilon) d \varepsilon}{\rho_{n}(E)}
$$

Here the sum is over all possible exciton states, which may be reached starting with a given initial exciton number. The residual nuclear excitation energy $U$ is given by $U=E$ $-(\varepsilon+B)$, where $B$ is the binding energy of the emitted particle.

The Griffin model was extended by Blann (1968) and others (Williams, 1970; Oblozinsky et al., 1974), and gives more complete expressions for the exciton-exciton transition rates [16].

$$
\begin{gathered}
\lambda_{n, n-2}=\frac{2 \pi}{\hbar}|M|^{2} g \frac{p h(n-2)}{2} \\
\lambda_{n, n}=\frac{2 \pi}{\hbar}|M|^{2} g^{2} E \frac{p(p-1)+4 p h+h(h-1)}{2 n} \\
\lambda_{n, n+2}=\frac{2 \pi}{\hbar}|M|^{2} \frac{g^{3} E^{2}}{2(n+1)}
\end{gathered}
$$


It is worth pointing out here that one significant difference between the exciton model and the hybrid model formulations is in the treatment of these transition rates. The hybrid and the geometry dependent hybrid models do not use the "matrix element" formulations (see next section).

After the preequilibrium emissions, the Hauser-Feshbach or Weisskopf-Ewing theories treat the remainder of the reaction process. A compact formula for the cross section for a reaction $\mathrm{A}(\mathrm{a}, \mathrm{b}) \mathrm{B}$ may be written as:

$$
\sigma_{a b}=\sum_{J \pi} \frac{T_{a} T_{b}}{\sum_{i} \sum_{c} T_{i c}}
$$

(where the index $i$ stands for the different types of outgoing particles (n,p,d,..) and the T's are the transmission coefficients calculated from an optical potential for particles $a$ and $b$. The index $c$ stands for all possible final states which are either discrete excited levels of the residual nucleus or a continuum of levels described by a level density formula. A recent handbook (IAEA TECDOC-1034, 1998) by IAEA is an excellent resource for information on optical potentials, various level density formulations and other aspects of modeling reaction cross sections.

Three groups and K. Gul (Islamabad, Pakistan) are involved in modeling cross sections for this CRP. The group at Obninsk used the Alice-IPPE code (Dityuk et al., 1998), the group from China used the Spec code (Shen and Zhang, 1993) and the Livermore group used HMS-Alice (Blann, 1996) for nucleon induced reactions and Alice-91 (Blann, 1991) for deuteron, ${ }^{3} \mathrm{He}$ and alpha induced reactions. The Alice family of codes are based on the hybrid, the geometry-dependent hybrid (GDH) or the HMS preequilibrium models and the Weisskopf-Ewing evaporation formalism. The Spec code is also constructed within the framework of the Weisskopf-Ewing evaporation formalism, but preequilibrium decay is calculated from the master equation exciton model (Blann, 1975). The HMS-Alice code uses a Monte Carlo precompound formulation with Weisskopf-Ewing evaporation. The lack of angular momentum and parity treatments in the Weisskopf-Ewing formalism used in these codes may be of some concern for certain aspects of reaction yields, (e.g., isomer yield calculations). But these codes are fast and convenient to use, i.e., when many natural isotopes are involved and many particles are emitted in the reaction process, and have generally been found to be adequate when cross 
sections for isomeric states are not needed. Gul used codes, HFMOD (Gul, 1995) and PREMOD (Gul, 1996), in his calculations. The HFMOD code is based on the HauserFeshbach formalism and the PREMOD code is based on the concept of the geometrydependent hybrid preequilibrium model generalized to include discrete levels, and to conserve angular momentum and parity. For complete formulations of the codes used in this CRP we refer to the appropriate references, but some of the highlights are given in the next section.

\subsection{Codes and calculations}

A variety of codes (Grogi, Stapre, Alice, Gnash, Spec, etc., and their modifications) have been developed on the basis of equilibrium and preequilibrium reaction mechanisms. These codes have similar physics with different degrees of complexity in input preparation and require different computing times. Some of them are used when detailed properties of nuclear reactions are needed, including population of discrete levels. For example, the Stapre and Gnash codes are good choices if one needs to have information on each channel participating in the reaction process, and when the excitation energy (and the number of open channels) is not too large. On the other hand, when the number of open channels is large and it is impossible or very time consuming to provide all the required input data with sufficient accuracy, the advantages of these detailed codes may be reduced. In such a case, the faster codes with less effort in input preparation are often more practical choices. The Alice family of codes developed by Blann and a recent modification by the Obninsk group fall in this class, and are used in the calculations of the reaction cross sections for medical radioisotope production for the present CRP. We refer to recent international code comparisons for further details on many of the codes in current use (Pearlstein, 1988; Blann et al., 1994; Michel and Nagel, 1997).

\section{Alice-91 code}

This is the latest released version of the standard Alice code at Livermore (Blann, 1991), which uses hybrid or geometry dependent hybrid precompound models and Weisskopf-Ewing evaporation for the equilibrium part of the reaction process. The basic physics has been widely described in the literature, but most physics can be found in (Blann, 1975; Blann and Vonach, 1983). Earlier versions of Alice did not include gamma 
ray competition with nucleon, deuteron and alpha emissions. The gamma competition is included since 1990. The gamma emission rate is calculated by microscopic reversibility based on the giant dipole with Lorentzian line shape (Blann et al., 1988). The Alice code uses the Fermi gas level density and has the option of using the shell-dependent level densities from Katatria and Ramamurthy (1980). The code includes multiple preequilibrium nucleon emission. Some of these features are described below.

\section{Hybrid model formulation}

The hybrid model (Blann, 1971) for precompound decay may be written as

$$
\frac{d \sigma}{d \varepsilon}=\sigma_{R} \sum_{n=n_{o}}^{\bar{n}}\left[\frac{X_{v}^{n} \rho_{n-1}(U)}{\rho_{n}(E)}\right]\left[\frac{\lambda_{c}(\varepsilon)}{\lambda_{c}(\varepsilon)+\lambda_{+}(\varepsilon)}\right] D_{n} d \varepsilon
$$

where the terms in the first set of brackets are the Ericson partial state densities, and $X_{v}^{n}$ is the number of excitons of type $v$ ( $v=$ neutron or proton) which are available for emission in the energy range $\varepsilon$ to $\varepsilon+d \varepsilon$, and $U=E-B_{v}-\varepsilon$, where $B_{v}$ is the binding energy of particle type $v$, neutron or proton. The term $\lambda_{c}(\varepsilon)$ is the rate of nucleon emission into the continuum, and $\lambda_{+}(\varepsilon)$ is the competing rate of two body collisions for the nucleons at energy $\varepsilon$. The factor $D_{n}$ is a depletion factor, which represents the fraction of the population surviving decay prior to reaching the $n$ exciton configuration.

The factor $X_{v}^{n}$ represents the exciton numbers for neutrons and protons for a given total exciton state $\mathrm{n}$. The default values in the Alice code for neutron induced reactions are

$$
X_{n}^{3}=\frac{2(3 Z+2 N)}{(3 Z+2 N+3 Z)}
$$

and

$$
X_{p}^{3}=2-X_{n}^{3}
$$

and for proton induced reactions

$$
X_{p}^{3}=\frac{2(3 N+2 Z)}{(3 N+2 Z+3 N)}
$$

and

$$
X_{n}^{3}=2-X_{p}^{3} .
$$


These choices are guided by the fact that for nucleon induced reactions the initial exciton state is a $2 p-1 h$ and that the $n-p$ scattering cross section is three times that of $n-n$ or $p-p$ scattering.

The corresponding values for deuteron and alpha induced reactions are 1,1 and 2,2. These initial values are assumed to increase by 0.5 in successive values of $n$ in Eq. (6), as the particle exciton number increases by 1 .

The nucleon-nucleon scattering rate is based on either the imaginary optical potential, where the mean free path is given by (Blann, 1973)

$$
\begin{gathered}
L(\varepsilon)=\left[\frac{\hbar^{2}}{4 m} \cdot \frac{1}{W^{2}}\left(\varepsilon+V+\sqrt{(\varepsilon+V)^{2}+W^{2}}\right)\right]^{1 / 2} \\
\cong \frac{\hbar^{2}}{2 m W} \sqrt{2 m / \hbar^{2}(\varepsilon+V)}
\end{gathered}
$$

or on Pauli-corrected nucleon-nucleon scattering cross sections, where the mean free path is given by (Kikuchi and Kawai, 1968)

$$
L(\varepsilon)=\frac{1}{\rho \bar{\sigma}_{0}},
$$

where $\rho$ is the density of nuclear matter and $\bar{\sigma}_{0}$ is the Pauli-corrected nucleon-nucleon $(\mathrm{N}-\mathrm{N})$ scattering cross section, appropriately weighted for target neutron and proton numbers. The intranuclear transition rate $\lambda_{+}(\varepsilon)$ is the quotient of nucleon velocity (in the potential well) divided by the mean free path. A closed form expression valid for nuclear matter of average density is given as (Blann, 1971)

$$
\lambda_{+}(\varepsilon)=1.4 \times 10^{21}\left(\varepsilon+B_{v}\right)-6 \times 10^{18}\left(\varepsilon+B_{v}\right)^{2} / \mathrm{sec},
$$

where $\varepsilon+B_{v}$ is the energy of nucleon $v$ above the Fermi energy. The continuum emission rate, $\lambda_{c}(\varepsilon)$ is given by microscopic reversibility as

$$
\lambda_{c}(\varepsilon)=(2 s+1) \Omega \frac{4 \pi p^{2} d p}{h^{3}} \bullet \frac{\sigma v}{\Omega g},
$$

where $s$ is the nucleon spin, $\Omega$ the laboratory volume, $p$ the nucleon momentum in the continuum, $g$ the single particle level density in the nucleus, $v$ the nucleon velocity in the laboratory, and $\sigma$ the inverse cross section. With these last two equations and the Ericson partial state density expression, we can calculate absolute PE spectra with Eq. (6). When we calculate N-N collision rates in the code ALICE, we have two options. One is to use 
the imaginary optical potential given by Becchetti and Greenlees (1969); the other is to use Eq. (9) calculating $\bar{\sigma}_{0}$ based on expressions due to Kikuchi and Kawai (1968) weighted for composite nucleus $\mathrm{N}$ and $\mathrm{Z}$ (rather than using the approximation of Eq. (10).

All the discussions above apply to a single precompound nucleon emission. Multiple nucleon emission, such as, $\mathrm{nn}, \mathrm{np}$ and $\mathrm{pp}$ emissions are also treated in the Alice -91 code (Blann and Vonach, 1983).

\section{Multiple particle emission}

Multiple precompound decay processes must be considered at higher excitations since they are important in determining the cross section surviving to the (equilibrium) compound nucleus, and in determining yields of products which require multiple precompound emission for population, e.g., a (p, 2p) reaction on a heavy element target. There are two types of multiple precompound decay which might be considered. Type I results when a nucleus emits more than one exciton from a single exciton hierarchy. It may be seen that, e.g., in a two-particle-one-hole configuration, up to two particles could be emitted; in a three-particle-two-hole configuration up to three particles could be emitted, etc.

The second type of multiple precompound decay (type II) would be described by the sequence "particle emission, one or more two body intranuclear transitions in daughter nucleus, particle emission." If the intervening two-body transitions are omitted from this sequence, it becomes type I multiple emission. In the type II sequence for nucleon induced reactions, the leading term would be two-particle-two-hole. Results confirm the speculation that type I multiple precompound decay is far more important than type II for most reactions at moderate excitations. Because the first particle emission leaves a range of residual excitations and exciton numbers, a calculation of type II emission becomes more complex and time consuming than for type I emission.

To extend Eq. (6) to higher energies and maintain its simplicity, we have made some arbitrary assumptions to estimate type I multiple particle emission branches. We define those assumptions which are based on simple probability arguments.

If $P_{n}$ and $P_{p}$ represents the total number of neutron and proton excitons emitted from a particular exciton number configuration, we assume that 


$$
P_{n p}=P_{n} P_{p}
$$

is the number of either type of particle emitted in coincidence with the other from the same nucleus and exciton hierarchy. This definition covers $P_{p n}$ since in an emission from the same exciton number there is no distinction to be made.

We assume that the number of neutrons which are emitted in coincidence with another neutron from a particular exciton number configuration is given by

$$
P_{n n}=2 \frac{P_{n}}{2} \frac{P_{n}}{2},
$$

with the fraction of the reaction cross section decaying by the emission of two coincident neutrons being $P_{n n} / 2$. The value of $P_{n n}$ is restricted to be $\leq P_{n}-P_{n n}$. Similar expressions are used for proton-proton coincident emissions.

The number of neutrons (protons) emitted from the n-exciton configuration, which is not in coincidence with another particle, is given by

$$
\begin{aligned}
& P_{n}(\text { n only })=P_{n}-P_{n n}-P_{n p} \\
& P_{p}(p \text { only })=P_{p}-P_{p p}-P_{n p}
\end{aligned}
$$

and the fraction of the population $F_{n}$ which had survived decay of the exciton number in question is

$$
F_{n}=1 .-P_{n}(n \text { only })-P_{p}(p \text { only })-P_{p p} / 2-P_{n n} / 2-P_{n p}
$$

This fraction would multiply the fractional population which had survived to the $n$ exciton state, i.e., is the depletion factor multiplier.

From the calculated total precompound neutron emission spectrum $d \sigma_{n}(\varepsilon) / d \varepsilon$, the cross section which could be involved in the emission of two neutrons is calculated as

$$
\sigma_{2 n}=\int_{U=0}^{E-B_{2 n}} \frac{d \sigma_{n}(\varepsilon)}{d \varepsilon} d \varepsilon
$$

where $B_{2 n}$ represents the sum of first and second neutron binding energies.

Similarly the neutron cross section which could be emitted in coincidence with protons is given by

$$
\sigma_{n p}=\int_{U=0}^{E-B_{n}-B_{p}} \frac{d \sigma_{n}(\varepsilon)}{d \varepsilon} d \varepsilon
$$


where $B_{n}$ is the binding energy of the first emitted neutron and $B_{p}$ is the proton binding energy of the daughter nucleus following neutron emission. Similar integrals are made for the proton emission cross section which could consist of two coincident protons, $\sigma_{p p}$, and of a proton in coincidence with neutron, $\sigma_{p n}$. The cross section available for the emission of a single nucleon $\sigma_{(p)}$ is, of course, the sum of all $d \sigma(\varepsilon) / d \varepsilon$ (the integrals are replaced by sums since the code computes spectra at fixed energy intervals). For the daughter nucleus following emission of one and only one precompound neutron, we calculate

$$
\sigma^{A-1, Z}(U)=\frac{d \sigma_{n}(\varepsilon)}{d \varepsilon} \frac{C_{n}}{\sigma_{n}}
$$

where $U-E-B_{n}-\varepsilon$; for the daughter nucleus following the coincident emission of two neutrons, we calculate

$$
\sigma^{A-2, Z}(U)=\frac{d \sigma_{n}(\varepsilon)}{d \varepsilon} \frac{C_{n n / 2}}{\sigma_{n n}}
$$

where $U=E-B_{2 n}-\varepsilon-\bar{\varepsilon}_{n}$, and where $\bar{\varepsilon}_{n}$ is the average kinetic energy of the second neutron for a given energy $\varepsilon$ of the first neutron. For the case of the daughter nucleus produced by the coincident emission of a neutron and a proton,

$$
\sigma^{A-2, Z-1}(U)=\frac{C_{n p}}{2 \sigma_{n p}}\left[\frac{d \sigma_{n}(\varepsilon)}{d \varepsilon}\right]+\frac{C_{n p}}{2 \sigma_{p n}}\left[\frac{d \sigma_{p}(\varepsilon)}{d \varepsilon}\right],
$$

where $U=E-B_{n}-B_{p}-\varepsilon-\bar{\varepsilon}_{p(n)}$ as previously defined, and where $\bar{\varepsilon}_{p(n)}$ is the average kinetic energy of the proton (neutron) emitted in coincidence with a neutron (proton) of kinetic energy $\varepsilon$. An expression analogous to Eq. (19) is used for the case of two-proton emission. The quantities $C_{n}, C_{p}, C_{n p}$ and $C_{n n}\left(C_{p p}\right)$ are defined as follows: $C_{n}$ is the cross section for emitting one and only one neutron summed over all exciton numbers, $C_{p}$ is the same as $C_{n}$ but for protons, $C_{n p}$ is for a neutron and a proton, and similarly for $C_{n n}$ and $C_{p p}$.

\section{Level densities and pairing options in Alice-91}

The Fermi gas level density used in the Alice codes is described first for completeness. The level density expression is 


$$
\rho(U) \propto(U-\delta)^{-5 / 4} \exp [2 \sqrt{a(U-\delta)}],
$$

where $U$ is the excitation energy and $\delta$ is the pairing gap. The level density parameter $a$ is given by, $a=A / 9$, as a default option. The pairing in Alice has two options: the backshift or a 'normal' shift with $\delta$ given by

$$
\delta=11 / A^{1 / 2} .
$$

The backshifted option uses true thermodynamic excitations for doubly even nuclei, and increases it by $\delta$ for odd A nuclei and $2 \delta$ for doubly odd nuclei. The normal shift option, on the other hand, uses true thermodynamic excitations for odd A nuclei, reduces the excitation by $\delta$ for doubly even nuclei, and increases it by $\delta$ for doubly odd nuclei. In addition, Alice has the option of using the $a$ and $\delta$ parameters from the Kataria and Ramamurthy formalism, which includes shell and pairing effects.

The Alice code has been used extensively and its predictive power is shown to be quite satisfactory up to $200 \mathrm{MeV}$ (Blann et al., 1994; Michel and Nagel, 1997). The Livermore group has done all their calculations for $d,{ }^{3} \mathrm{He}$, and alpha induced reactions for this CRP using the hybrid PE formulation. (They used the Monte Carlo approach as in HMS-Alice for nucleon induced reactions).

\section{Geometry Dependent Hybrid Model (GDH)}

The basic ideas are described here (Blann, 1972): The nucleus has a density distribution which can affect PE decay in two ways. First, the nucleon mean free path is expected to be longer (on average about a factor of two) in the diffuse nuclear surface. Secondly, in a local density approximation, there is a density dependent limit to the hole depth; this will be expected to additionally modify the Ericson state densities with respect to use of a single, averaged potential depth. These two changes were incorporated into the 'geometry dependent hybrid model'. We present next a description of these changes with specific reference to the code Alice. (The Obninsk group uses the GDH formulation option of Alice in their Alice-IPPE code with some modifications, which are described later.)

In order to provide a first order correction for the influence of nuclear density, the hybrid model may be reformulated as a sum of contributions, one term for each entrance channel impact parameter with parameters evaluated for the average local density of each impact parameter. In this way, the diffuse surface properties sampled by the higher 
impact parameters are crudely incorporated into the precompound decay formalism in the GDH model. The differential emission spectrum is given in the GDH model as

$$
\frac{d \sigma_{v}(\varepsilon)}{d \varepsilon}=\pi \hbar^{2} \sum_{\ell=0}^{\infty}(2 \ell+1) T_{\ell} P_{v}(\ell, \varepsilon),
$$

where $\ell$ is the entrance channel orbital angular momentum, $T_{\ell}$ the transmission coefficient and $P_{v}(\ell, \varepsilon)$ is the decay probability at exit channel energy $\varepsilon$, as given in Eq. (6) . When the approach is used for incident nucleons, $T_{\ell}$ is provided by an optical model subroutine. Whereas the intranuclear transition rates entering in the hybrid model are evaluated for nuclear densities averaged over the entire nucleus, those appropriate for the GDH model are averaged over the densities corresponding to the entrance channel trajectories, at least for the contributions from the first projectile-target interaction. The multi-particle preequilibrium emission is treated in the GDH model as it is described in the hybrid model.

The effect of limited hole depth is less physically established than the influence of density on mean free path; nonetheless it seems to be important in reproducing experimental spectral shapes. The result of this local density approximation limitation is to effectively reduce the degrees of freedom, especially for the higher incident partial waves (for which a lower maximum hole depth is predicted), thereby hardening and enhancing the predicted emission spectra. In our use of Alice, we use the option under which the restriction on hole depth in the GDH model applies only to the first collision, for which there is some knowledge of average density at the collision site, and then only for nucleon induced reactions.

The original GDH model employed a Fermi density distribution function,

$$
d\left(R_{\ell}\right)=d_{s}\left[\exp \left(R_{\ell}-C\right) / 0.55 f m+1\right]^{-1}
$$

with

$$
C=1.07 A^{1 / 3} \mathrm{fm},
$$

taken from electron scattering results (Hofstadter, 1957). The radius for the $\ell$ th partial wave was defined by

$$
R_{\ell}=\lambda(\ell+1 / 2)
$$


The charge radius $C$ of Eq. (25) has been replaced in the present parameterization by a value characteristic of the matter (rather than charge) radius based on the droplet model work of Myers (1977), plus a projectile range parameter $\lambda$,

$$
C=1.18 A^{1 / 3}\left[1-1 /\left(1.18 A^{1 / 3}\right)^{2}\right]+\lambda .
$$

In the hybrid model, the average nuclear density is calculated by integration and averaging of Eq. (27) between $R=0$ and $R=C+2.75 \mathrm{fm}$. The single particle level densities are defined in the precompound routine of Alice by

$$
g_{n}=\frac{N}{28}, \text { and } g_{p}=\frac{Z}{28} .
$$

\section{HMS-Alice code}

The HMS-Alice code is based on a new precompound Monte Carlo simulation (HMS) model described in (Blann, 1996). The model does not rely upon exciton state densities beyond three excitons, permits unlimited multiple precompound emission for each interaction and may be used to calculate exclusive particle spectra and yields. The evaporation part of the calculation is done with the usual Weisskopf-Ewing formalism, as in the Alice-91 code.

The HMS precompound decay model is formulated to reduce several inconsistencies and limitations of earlier formulations, such as in the hybrid, GDH and exciton models. These precompound formulations have relied upon contributions from the entire exciton populations based on a sequence of two-body collisions. It is further assumed that all possible ways of sharing the excitation energy between different particle-hole configurations with the same exciton number have equal a-priori probability. It was clearly shown the equal a-priori population assumption may be valid only for states with the first exciton number (three excitons for nucleon induced reactions) (Blann and Vonach, 1983) and not for higher exciton states (Bisplinghoff, 1986). Additionally, existing precompound formulations were not suited for multiple PE emissions beyond two, yet this becomes important at energies above $\sim 50 \mathrm{MeV}$. To overcome these problems, Blann developed the HMS Monte Carlo prcompound model,which uses only the kinematically justified two and three exciton densities with unlimited precompound particle emission. The formulation otherwise follows the 
philosophy of the hybrid model. The new approach is therefore referred to as the hybrid Monte Carlo simulation (HMS) model. It should be valid up to the pion threshold.

In the HMS approach, incident nucleons that make only 3-exciton states are considered. For each nucleon energy (selected at weighted random from the possible range of energies) the nucleon will either be emitted, or will rescatter. If it is emitted, the emission probability is calculated from equations (6), (9), and (11) of the hybrid model, using the $n=n_{0}$ term only in Eq. (6). (The remaining two quasiparticles will share the balance of the excitation energy and will initiate their own 3-quasiparticle states and then either decay or rescatter.) If the initial nucleon rescatters, it will make a new 3-exciton state and the process of decy or rescatter continues. The hole energy is similarly allowed to initiate a 2 hole-1particle state, etc. In this fashion each cascade treats only the physically justified 2 and 3 exciton states, and can treat any number of precompound decays for each cascade.

The HMS model enjoys another advantage over closed form decay models (e.g., exciton, hybrid or GDH models) for calculation of particle spectra and recoil distributions. Because it is preformed in an event mode, the velocity of the emitting nuclide may be modified according to the angle and energy of each nucleon previously emitted, giving proper laboratory/center of mass transformations. The two-body assumption necessary in closed form calculations may be seen to be quite poor when comparisons are made between the two models.

The Monte Carlo precompound formulation is available at present for neutron and proton induced reactions, but not for deuteron, ${ }^{3} \mathrm{He}$ or alpha particle induced reactions. The Livermore group therefore used HMS-Alice for proton induced reactions in this CRP, but used the Alice-91 code for all other reactions. Both codes used optical models for incident nucleons, and for all inverse reaction cross sections. The parameter sets used are described in (Blann and Vonach, 1983).

\section{Livermore calculations}

The calculations by the Livermore group for this CRP are based on global optical potentials included in the code, and two level density options: Fermi gas level density or shell dependent level densities given by the model of Kataria and Ramamurthy (1980). For deuteron induced reactions Livermore studied the effects of the deuteron breakup in 
the entrance channel (Mustafa, 1977) using a microscopic theory developed by Udagawa and Tamura (1981 and 1986) coupled with a modified Alice-91 code. In this approach the Udagwa and Tamura model was used to deduce the spectra of neutrons and protons transferred into the target nucleus (stripping to bound states and breakup-capture in the continuum); these were then assumed to initiate three-quasiparticle cascades. The deuteron cross section that does not undergo stripping and breakup-capture was assumed to initiate a separate preequilibrium cascade. Detailed calculations were done for deuteron induced reactions on ${ }^{48} \mathrm{Ti}$ in order to empirically select the best initial exciton number to use for deuteron induced reactions. The results were used to choose the precompound initial exciton number parameters for the remaining deuteron induced reactions for this CRP. The total number of initial excitons thus chosen in the calculation is two, i. e., one proton and one neutron above the Fermi surface. The ${ }^{3} \mathrm{He}$ induced reactions are also known to have a sizeable breakup cross section. This problem has not been addressed here. Therefore, the choice of the initial exciton number for ${ }^{3} \mathrm{He}$ induced reactions and also for alpha induced reactions is arbitrary. Four excitons, two protons and 1 neutron (and one hole) are used for ${ }^{3} \mathrm{He}$ induced reactions, and four excitons, two protons and two neutrons, for alpha induced reactions. Because of the global nature of these parameters, the calculated cross sections for this CRP should be used as a guide rather than as a fit to the experimental data. Using a single global parameter choice allows an estimation of the predictive powers of the models; varying parameters to fit each data set precludes this.

\section{Alice-IPPE code}

The Alice-IPPE code is the Alice-91 code version modified by the Obninsk group (Dityuk et al., 1998) to include the generalized superfluid level density model of Ignatyuk and colleagues (Ignatyuk, 1983; Ignatyuk et al., 1979; Blokhin et al., 1988; Ignatyuk et al., 1993) and preequilibrium cluster emissions. For the preequilibrium nucleon emission the geometry dependent hybrid (GDH) model is used. Calculation of the alpha-particle spectra is performed taking into account both the pickup (Iwamoto and Harada, 1982; Sato et al., 1983) and knockout processes (Milazzo-Colli and BragaMarcazzan, 1973; Ferrero et al., 1979; Oblozinsky and Ribansky, 1978). A phenomenological approach is used to describe direct emission of the deuteron (Dityuk et al., 1998). The triton and ${ }^{3} \mathrm{He}$ spectra are calculated according to the coalescence pickup 
model of Sato, Iwamoto and Harada (1983). The level density formalism includes both collective and non-collective effects, and excitation-energy-dependent shell effects. These level density improvements over the Fermi gas model are described here. For details on the cluster emission models and calculations we refer to (Dityuk et al., 1998) and references therein.

\section{Generalized superfluid model of level densities}

The Fermi gas model, as used in the Alice-91 and HMS-Alice codes, has a simple and convenient form for global applications. However, the model is inadequate when nuclear shell and collective effects become important. (A simple approach due to Kataria and Ramamurthy to incorporate shell structure effects in the level densities has been mentioned earlier and offered as an option in the Alice codes.) Detailed microscopic calculations are now possible, but they are time consuming and their success in reproducing experimental level densities are yet to be proven. Ignatyuk and his colleagues at Obninsk have developed a phenomenological approach to level density calculations which includes both shell effects and collective enhancements (rotational and vibrational) to level densities. This is referred to as the generalized superfluid model and has been incorporated in the Alice-IPPE code.

The level density expression has now three components:

$$
\rho(U)=\rho_{q p}\left(U^{\prime}\right) K_{v i b}\left(U^{\prime}\right) K_{r o t}\left(U^{\prime}\right)
$$

where $\rho_{q \rho}\left(U^{\prime}\right)$ is the level density due to quasiparticle (non-collective) nuclear excitations only, and $K_{v i b}\left(U^{\prime}\right)$ and $K_{r o t}\left(U^{\prime}\right)$ are the level density enhancements due to vibrational and rotational states, respectively, at the effective excitation energy $U^{\prime}$.

The energy dependence of the quasiparticle level density is calculated on the basis of the superfluid nuclear model (Ignatyuk et al., 1979). The correlation function for the ground states of nuclei is defined as $\Delta_{0}=12.0 / A^{1 / 2} \mathrm{MeV}$. This choice of $\Delta_{0}$ is consistent with the systematics of nuclear masses (Myers, 1977) and with analysis of the experimental data on neutron resonances (Ignatyuk et al., 1979). The critical temperature of the phase transition from superfluid to normal state, the condensation energy, the critical energy of the phase transition and the effective excitation energy are connected with the correlation function $\Delta_{0}$ by the following equations: 


$$
\begin{gathered}
t_{c r}=0.567 \Delta_{0} \\
U_{c r}=0.472 a_{c r} \Delta_{0}^{2}-n \Delta_{0} \\
E_{c o n}=0.152 \cdot a_{c r} \Delta_{0}^{2}-n \Delta_{0} \\
U^{\prime}=U+n \Delta_{0}+\delta_{\text {shift }},
\end{gathered}
$$

where $n=0,1$ and 2 for even-even, odd and odd-odd nuclei, respectively, and the value of the excitation energy shift $\delta_{\text {shift }}$ is chosen on the basis of a consistent description of the level density of low lying collective levels and the data on neutron resonances.

The shell effects are included into consideration using the energy dependence of nuclear level density parameter $a(U, A)$, determined phenomenologically:

$$
\begin{gathered}
a(U, Z, A)=\tilde{a}(A)\left(1+\delta W(Z, A) \frac{\varphi\left(U^{\prime}-E_{\text {cond }}\right)}{U^{\prime}-E_{\text {cond }}}\right), \quad U^{\prime}>U_{c r} \\
a(U, Z, A)=a\left(U_{c r}, Z, A\right), \quad U^{\prime}<U_{c r}
\end{gathered}
$$

where the asymptotic value of level density parameter at high excitation energy is equal to

$$
\tilde{a}(A)=0.073 A+0.115 A^{2 / 3},
$$

and where $\delta W(Z, A)$ is the shell correction to the nuclear binding energy taken from the experimental values of nuclear masses or from the Myers-Swiatecki mass formula (1967) when experimental masses are unknown. The function $\varphi(U)=[1-\exp (-\gamma U)]$ is dimensionless and defines the energy dependence of the level density parameter at low excitation energies within the value of $\gamma=0.4 / A^{1 / 3}$ chosen from the neutron resonances.

The vibration enhancement of nuclear level density is presented in the following form

$$
K_{v i b}=\exp [\delta S-(\delta U / t)]
$$

where $\delta S$ and $\delta U$ are the changes in the entropy and excitation energy, respectively, resulting from the vibrational modes. These changes are described by the relations of the Bose gas:

$$
\delta S=\sum_{i=1}^{\infty}\left(2 \lambda_{i}+1\right)\left[\left(1+n_{i}\right) \ln \left(1+n_{i}\right)-n_{i} \ln \left(n_{i}\right)\right]
$$




$$
\delta U=\sum_{i=1}^{\infty}\left(2 \lambda_{i}+1\right) \omega_{i} n_{i}
$$

where $\omega_{i}$ and $\lambda_{i}$ are the energies and the multipolarities of collective excited state, and $n_{i}$ is its population at a given temperature. The attenuation of vibrational enhancement of level density at high temperatures is taken into account with the following occupation number dependence:

$$
n_{i}=\frac{\exp \left[-\gamma_{i} /\left(2 \omega_{i}\right)\right]}{\exp \left[\omega_{i} / t\right]-1}
$$

where $\gamma_{i}$ is the spreading width of the vibrational excitation and is given by

$$
\gamma_{i}=0.0075 A^{1 / 3}\left(w_{i}^{2}+4 \pi^{2} t^{2}\right)
$$

The quadrupole and octupole states are considered in the calculations only. The position of the lowest state for the all nuclei, with exception of ${ }^{208} \mathrm{~Pb}$, was defined by phenomenological equations which reproduced the experimental data well enough for middle weight nuclei:

$$
\omega_{2}=30 A^{-1 / 3}, \quad \omega_{3}=50 A^{-1 / 3} .
$$

For all spherical nuclei the coefficient of vibrational enhancement of the level $K_{\text {vibr }}\left(U^{\prime}\right)$ was taken into account according to Eq. (33). For deformed nuclei the enhancement of level density connected with the rotational mode of collective excitation $K_{r o t}\left(U^{\prime}\right)$ was taken into account according to Bohr and Mottelson (1974):

$$
K_{r o t}(U)=\sigma_{\perp}^{2} g(U)=\sigma^{2}(1+\beta / 3) g(U),
$$

where $\sigma^{2}$ is the spin cut-off factor, and $g(U)$ is the empirical function taking into account the attenuation of rotation modes at high energies as proposed by Hansen and Jensen (1983):

$$
g(U)=\left[1+\exp \left[\left(U-U_{r}\right) / d_{r}\right]\right]^{-1},
$$

where the parameters of the attenuation function are connected with the quadrupole nuclear deformation $\beta$ by the relations:

$$
U_{r}=120 A^{-1 / 3} \beta^{2} ; d_{r}=1400 A^{-2 / 3} \beta^{2} .
$$

The parameter $\beta$ is taken from Myers (1977). 
We have described above the important features of the level density calculations using the generalized superfluid model of Ignatyuk and colleagues, as included in the Alice-IPPE code. These improvements over the Fermi gas model description of the level density are based on components of the nuclear structure theory, i.e., pairing, shell and collective effects.

\section{Spec Code}

SPEC (Shen and Zhang, 1993) is a program for calculating the neutron or charged particle $\left(p, d, t,{ }^{3} \mathrm{He}, \alpha\right)$ induced reactions on medium-heavy nuclei in the incident energy range up to $60 \mathrm{MeV}$ including up to 6 successive emission processes per nucleus. For those reaction channels contributed only by $1 \sim 5$ emission processes the incident energy can go up to $100 \mathrm{MeV}$. This program is written in FORTRAN-77.

SPEC is constructed within the framework of the optical model, the master equation exciton model (Blann, 1975), and the Weisskopf-Ewing evaporation model (1940). For the first and second particle emission processes, the preequilibrium emission and evaporation are considered, but for 3-6 particle emission processes, only evaporation is considered. The preequilibrium and direct reaction mechanisms of $\gamma$ emission (Akkermans et al., 1985) are also included. The effect of the recoil nucleus is considered for calculating spectra.

The master equation exciton model is given by

$$
\begin{aligned}
-\delta_{n, n_{0}}= & \lambda_{-}(n+2, E) \tau(n+2, E)+\lambda_{+}(n-2, E) \tau(n-2, E) \\
& -\left[\lambda_{+}(n, E)+\lambda_{-}(n, E)+W_{\ell}(n, E)\right] \tau(n, E),
\end{aligned}
$$

where $\mathrm{E}$ is the excitation energy. The quantities $\gamma_{+}$and $\gamma_{-}$are the intranuclear transition rates. For composite particle emission, the pick-up mechanism of cluster formation (Iwamoto et al., 1982; Sato et al., 1983; Zhang et al., 1988; Zhang et al., 1992) is used in the first and second particle emission processes. The cluster $b$ is defined by the distribution of particles above and below the Fermi surface $(\ell, m)$. By means of the detailed balance principle the emission rate of $\ell$ particles above Fermi surface can be expressed as

$$
W_{b}^{\ell}\left(p, h, E, \varepsilon_{b}\right)=\frac{2 I_{b}+1}{\pi^{2} h^{3}} \mu_{b} \varepsilon_{b} \sigma_{b}\left(\varepsilon_{b}\right) F_{\ell, m}\left(\varepsilon_{b}\right)
$$




$$
Q_{\ell, m}^{b}(p, h) \frac{\rho\left(p-\ell, h, E-\varepsilon_{b}-B_{b}\right)}{\rho(p, h, E)},
$$

where $I_{b}$ is the spin of the emitted particle $b, \rho$ is the exciton state density, $\sigma_{b}\left(\varepsilon_{b}\right)$ is the inverse cross section of the emitted particle $b$ with outgoing energy $\varepsilon_{b}, B_{b}$ is the binding energy of particle b in the system, $Q_{\ell, m}^{b}(p, h)$ is a combination factor and $F_{l m}\left(\varepsilon_{b}\right)$ is the pick-up factor of the emitted particle.

The emission rate for a photon with energy $\varepsilon_{\gamma}$ from a nucleus in the exciton state $n$ is taken as (Akkermans et al., 1985):

$$
W_{\gamma}\left(n, \varepsilon_{b}\right)=\frac{\varepsilon_{\gamma}^{2} \sigma_{a}^{\gamma}\left(\varepsilon_{\gamma}\right)}{\pi^{2} h^{3} C^{2} \rho_{n}(E)}\left\{\frac{g^{2} \varepsilon_{\gamma} \rho_{n-2}\left(E-\varepsilon_{\gamma}\right)}{g(n-2)+g^{2} \varepsilon_{\gamma}}+\frac{g n \rho_{n}\left(E-\varepsilon_{\gamma}\right)}{g n+g^{2} \varepsilon_{\gamma}}\right\},
$$

where $g$ is the single particle level density. $\sigma_{a}^{\gamma}\left(\varepsilon_{\gamma}\right)$ is the giant resonance cross section:

$$
\sigma_{a}^{\gamma}\left(\varepsilon_{\gamma}\right)=\sum_{i=1}^{2} \frac{\left(\varepsilon_{\gamma} \Gamma_{i}\right)^{2} \sigma_{i}^{\gamma}}{\left(\varepsilon_{\gamma} \Gamma_{i}\right)^{2}+\left(\varepsilon_{\gamma}^{2}-E_{i}^{2}\right)^{2}}
$$

where $\Gamma_{i}, E_{i}$ and $\sigma_{i}^{\gamma}$ are two peak giant resonance parameters.

For the first emission of particle $b$, the spectrum is given by

$$
\frac{d \sigma_{b}}{d \varepsilon_{b}}=\sigma_{a} \sum_{p n} \tau(p, h, E) \sum_{\ell} W_{b}^{\ell}\left(p, h, E, \varepsilon_{b}\right),
$$

where $\sigma_{a}$ is the absorption cross section of the incident particle. The direct $\gamma$ emission cross section is

$$
\sigma_{\gamma}^{d}=\sigma_{a} \frac{Y}{Y+\lambda_{+}(1)}
$$

where

$$
\begin{gathered}
\lambda_{+}(1)=\frac{\pi K}{2}\left(\frac{g}{A}\right)^{3} E \\
Y=\frac{1}{\pi^{2} h^{3} C^{2}} \int_{0}^{E} \varepsilon_{\gamma}^{2} \sigma_{a}^{\gamma}\left(\varepsilon_{\gamma}\right) \frac{1}{1+g \varepsilon_{\gamma}} d \varepsilon_{\gamma},
\end{gathered}
$$

where $K$ is the exciton model constant and $\mathrm{A}$ is the mass number of the composite system. 
The Gilbert-Cameron level density formula (1965) was used in the program SPEC. The inverse cross sections of the emitted particles used in statistical theory are calculated from the optical model. The partial widths for $\gamma$-ray emission are calculated based on the giant dipole resonance model with two resonance peaks in both the evaporation model and the exciton model.

In the optical model calculation, the Becchetti and Greenlees (1969) phenomenological optical potentials are used. The Neumanove methods are used to solve the radial equation of the optical model. Coulomb wave functions are calculated by the continued fraction method (Barnett et al., 1974).

The following nuclear data can be calculated by using the program SPEC: total emission cross sections and spectra of all emitted particles; the partial emission cross sections and spectra of all emitted particles from the first to sixth particle emission processes and different pick-up configurations $(1, \mathrm{~m})$; the various yield cross sections; total and elastic scattering cross sections (only for neutron as projectile); total reaction cross section; nonelastic scattering cross sections; radiative capture cross section; (x,np), $(\mathrm{x}, \mathrm{n} \alpha),(\mathrm{x}, 2 \mathrm{n}),(\mathrm{x}, 3 \mathrm{n}),(\mathrm{x}, 4 \mathrm{n}),(\mathrm{x}, 5 \mathrm{n}),(\mathrm{x}, 6 \mathrm{n})$ cross sections and so on. SPEC can not be used to calculate the direct inelastic scattering and compound elastic scattering cross sections. The applications so far show that the SPEC is a useful and convenient code for users.

\section{GUL Calculations}

Gul used the HFMOD Code (Gul, 1995) for statistical model and PREMOD Code (Gul, 1996) for preequilibrium model nuclear reaction calculations. The calculations from the two codes were combined after correcting the Hauser-Feshbach calculation for flux reduction in the preequilibrium mode.

The HFMOD Code is based on the Hauser-Feshbach formalism (1952). The angle-integrated cross section from channel $n$ to channel $m$ is given by

$$
\sigma_{n m}=\frac{\pi \hbar^{2}}{\left(2 i_{n}+1\right)\left(2 I_{n}+1\right)} \sum_{J P}\left[\frac{(2 J+1) \sum_{\ell \ell^{\prime} j j^{\prime}} T_{\ell}^{n} T_{\ell^{\prime}}^{m}}{\sum_{\ell j^{\prime \prime} q^{\prime \prime}} T_{\ell^{\prime \prime}}^{q^{\prime \prime}}}\right] \text {, }
$$


where $\lambda$ is the rationalized wavelength of the particle of spin $i_{n}$ incident on the target nucleus of $\operatorname{spin} \mathrm{I}_{\mathrm{n}} ; \ell$ and $j$ are the orbital angular momentum of the incoming particle and incident channel spin, and $l^{\prime}$ and $j^{\prime}$ are the orbital angular momentum of the outgoing particle and exit channel spin. $\mathrm{J}$ and $\mathrm{P}$ specify summation over all possible spin and parity values of the compound nucleus; $q$ " specifies summation over all possible competing channels which include particle and gamma emission, implying integration over the continuum states of the residual nuclei weighted by appropriate values of level densities. $T_{\ell}^{n}$ and $T_{\ell^{\prime}}^{m}$ are the transmission coefficients of the incident and outgoing particles which are calculated using appropriate optical model parameters. The code has options for the calculation of level densities on the basis of the Gilbert-Cameron composite formula (1965) or the formalism of Dilg et al. (1973). In the present calculations the energy level densities were computed using the formalism of Dilg et al. The competition from photon emission has also been included in the second stage while considering emission of a particle from the residual nuclei. Angular momentum and parity are conserved in both stages of the calculation. Transmission coefficients for photon emission are calculated on the basis of the single-particle model of Aslam Lone (1978) and Wilkinson (1970). (An option to use the Brink-Axel formalism for E1 emission is also available in the code.) The single-particle energy level spacing is used as an adjustable parameter in all the calculations. Perey potentials (1963) are used for protons and Wilmore-Hodgson potentials (1974) are used for neutrons. Averageanu et al. potentials (1994) are used for alpha-particles and Vernotte et al. potentials (1982) are used for helions.

\section{PREMOD Code}

The pre-equilibrium contribution to nuclear reactions is calculated using the PREMOD Code (Gul, 1996). This code is based on the concept of the geometrydependent hybrid model generalized to include discrete levels, and to conserve angular momentum and parity. The cross section per level is given by

$$
\sigma_{v}(E, U)=\frac{\pi \hbar^{2}}{(2 S+1)(2 I+1)} \sum_{J P}(2 J+1) \sum_{n} \sum_{\ell} T_{l}^{J} \sum_{\ell^{\prime}}\left[X_{v}^{n} \frac{\rho\left(p-1, U, J^{\prime}\right)}{\rho(p, h, E, J)}\right] \times
$$




$$
\frac{\left(\frac{1}{2 \pi \hbar g}\right)\left(2 S_{v}+1\right)(2 \ell+1) T_{\ell^{\prime}}^{J}}{\sum_{q} \sum_{\ell^{\prime}}\left(\frac{1}{2 \pi \hbar g}\right)\left(2 S_{v}+1\right)(2 \ell+1) T_{\ell^{\prime}}^{J}+\lambda^{+}} D_{n}^{J P},
$$

where $\lambda$ is the rationalized wave length of the incident particle of spin $S$ incident on the target of spin $I . J$ and $P$ are spin and parity of the composite state; $n$ is the exciton number, which is equal to the sum of particle number $\mathrm{p}$ and hole number h. $T_{\ell}^{J}$ and $T_{\ell^{\prime}}^{J}$ are the transmission coefficients of the incident and outgoing particles corresponding to partial waves $\ell$ and $\ell^{\prime} ; X_{v}^{n}$ is the number of excitons of type $v$ (protons or neutrons) in the n-exciton state. $\rho(p, h, E)$ is the state density of n-exciton configurations of the composite state characterized by excitation energy $E$, particle number $p$ and hole number $h(n=p+h)$. $\rho(p-1, h, U)$ is the state density of the residual nucleus. $D_{n}^{J P}$ is a depletion factor that takes into account the depletion of the flux due to the reaction taking place from exciton states characterized by exciton number less than n. $S_{v}$ is the spin of the outgoing particle of type $v . q$ specifies the sum over available channels for the emission of particle $v$ from the composite state to the discrete states, as well as states of constant density $g$ in the continuum of the residual nucleus. The spin-dependent level density is calculated as recommended by Feshbach et al. (1980).

$$
\rho(n, E, J)=\rho(n, E) \quad R(E, n, J),
$$

where

$$
\rho(n, E)=\frac{g^{n} E^{n-1}}{p ! n !(n-1) !}
$$

The excitation energy $E$ is corrected for the Pauli effect by replacing it with $E^{\prime}$, where

$$
E^{\prime}=E-P(h, p),
$$

and

$$
P(h, p)=\frac{p^{2}+h^{2}+p-3 h}{4 g} .
$$

The spin-dependent factor $\mathrm{R}(\mathrm{E}, \mathrm{n}, \mathrm{J})$ is taken as 


$$
R(E, n, J)=\frac{2 J+1}{\pi^{1 / 2} n^{3 / 2} \sigma^{3}} \exp \left[-\frac{(J+0.5)^{2}}{n \sigma^{2}}\right],
$$

where the spin-cutoff parameter $\sigma$ is given by

$$
\sigma=\left(\frac{2 c \tau}{\bar{n}}\right)^{1 / 2}
$$

where $\tau$ is nuclear temperature given by

$$
E=a \tau^{2}-\tau \quad \text { with } \quad a=\frac{\pi^{2}}{6} g .
$$

The following values as suggested by Feshbach et al. (1980) are used.

$$
a=A / 8 \mathrm{MeV}^{-1} \quad g=\left(\frac{6}{\pi^{2}}\right) A / 8 \mathrm{MeV}^{-1} \quad c=\frac{A^{5 / 3}}{90} \mathrm{MeV}^{-1} .
$$

$\bar{n}$ is the average exciton number equal to $1 / 2(2 g E)^{1 / 2}$. Particle emission is considered in competition with internal cascade transitions leading only to higher exciton number. The transition probability $\lambda^{+}$from the state of exciton number $n$ to $n+2$ is equal to $2 \mathrm{~W} / \hbar$ (Kikuchi and Kawai, 1968), where W is the imaginary component of the optical model potential. The probability of existence $X_{v}^{n}$ of particle $v$ in n-exciton state can be calculated for neutron and proton induced reactions in which a nucleon is emitted, as described earlier for the Alice code.

For nucleon induced reactions in which an alpha-particle is emitted, the concept of the preformed alpha-particle is assumed and the values of $X_{v}^{n}$ are calculated as described by Gadioli et al. (1977) and Milazzo-Colli and Braga-Marcazzn (1973).

$$
X_{a}^{n}=\frac{\phi K_{a}^{p-1, h}}{\phi K_{a}^{p, h}+(1-\phi) K_{n}^{p, h}},
$$

where $K_{a}^{p, h}$ is a coefficient which represents the percentage of states containing an excited $\alpha$-particle and $\alpha$-hole in the state level density corresponding to $\mathrm{p}+\mathrm{h}$ excitons. $K_{a}^{p, h}$ is the coefficient representing the percentage of states of nucleons and nucleonholes in the state level density corresponding to $\mathrm{p}+\mathrm{h}$ excitons. The coefficient $\phi$ represents the probability that a neutron or proton interacts with a preformed alpha particle. In fact it is a normalization parameter and its values lie in the $0.5-1$ range. The 
optical model parameters used for Hauser-Feshbach calculation are also used for preequilibrium calculations. The initial exciton number for nucleon, alpha and helion is taken as 3,4 , and 5 , respectively.

\section{Chapter 3.3 Discussion of the modeling results}

The codes used by the four different modeling groups for this CRP are described in the previous section. These codes are Alice-91 and HMS-Alice (Livermore), AliceIPPE (Obninsk), SPEC (Beijing), and HFMOD and PREMOD (Islamabad). In this section, we report modeling results for selected reactions to highlight the successes and failures of the modeling efforts by the four groups in reproducing the experimental data. (Comparison of the modeled cross sections with experimental data for all the reactions in the CRP is shown in the next section.) The calculations are based on global input parameters for optical potentials and level densities. The calculations start with the preequilibrium emissions followed by the evaporation. The preequilibrium models used are hybrid or geometry dependent hybrid (GDH) by all the codes, except for the SPEC and the HMS-Alice, which use the master equation exciton model and Monte Carlo nucleon emissions, respectively. The evaporation is calculated according to the Weisskopf-Ewing model, except for the HFMOD code where the Hauser-Feshbach formalism is used. In terms of the level density descriptions, the Livermore group used the Fermi gas model level densities and the level densities from Kataria and Ramamurthy (1980) with shell effects. The Obninsk group used level densities from the generalized superfluid model, which includes shell effects and collective enhancements. The Beijing group used the Gilbert-Cameron level densities and Gul (Islamabad) used backshifted Fermi gas model parameters of Dilg et al. Cluster preequilibrium emissions are included in the Alice-IPPE calculations.

Because of the global nature of the input parameters, the calculated cross sections are used mostly as a guide rather than a fit to the experimental data, although in some cases modeled cross sections were chosen as the recommended values by this CRP. In general the calculated cross sections show a better fit to the data for $\mathrm{A}>30$, and also

show a better fit for proton induced reactions over deuteron, ${ }^{3} \mathrm{He}$ and alpha. The poor fit to the lower mass targets may be attributed to the reaction mechanism used in the codes. It is known that nuclear structure plays a critical role in the lighter masses and that the R- 
matrix theory is more appropriate in modeling cross sections for such targets. Because of the complexity in modeling such cross sections, it was decided by the CRP not to pursue the modeling for light targets but to use only the experimental data for the evaluation. Another important modeling feature that was not explicitly included is the breakup of light projectiles, such as, the deuteron and ${ }^{3} \mathrm{He}$, in the entrance channel of the reaction process. However, the Livermore group made their choice of the preequilibrium model parameters, namely the initial exciton numbers, from an investigation of the deuteron breakup (i.e., the stripping to the bound states and breakup-capture in the continuum) for $\mathrm{d}+{ }^{48} \mathrm{Ti}$ reaction. The Obninsk group reduced their calculated cross sections by a factor of 1.5 for deuteron induced reactions to account for such breakup processes.

In Fig. 1 we show the effects of the deuteron breakup in ${ }^{48} \mathrm{Ti}(\mathrm{d}, 2 \mathrm{n})^{48} \mathrm{~V}$ cross sections calculated by the Livermore group using the Udagawa and Tamura (1981 and 1983) microscopic theory. In their approach the Udagwa and Tamura theory was used to deduce the spectra of neutrons and protons transferred into the target nucleus (stripping to bound states and breakup-capture in the continuum); these were then assumed to initiate three-quasiparticle preequilibrium cascade and evaporation. The deuteron cross section that does not undergo stripping or breakup-capture was assumed to initiate a separate preequilibrium cascade and evaporation. In the figure the "breakup" refers to the sum of cross sections from these two processes, i.e., the breakup and also the remaining cross sections coming from the optical model reaction cross section that did not undergo breakup. The three other curves, identified by 6-2-2, 3-1-1 and 2-1-1, are Alice-91 calculations with no breakup. The numbers, e.g., 6-2-2, etc., are the initial exciton numbers (total, neutron and proton excitons). These calculations were done in order to empirically select the best initial exciton numbers to use for the deuteron induced reactions, when breakup can not be included explicitly. The best fit to the data near the peak cross section is found when the breakup is included. The calculation with 2-1-1 is closer to the breakup model calculation and the data. However, note that even with these choices of the initial exciton numbers the calculated cross sections are about a factor of two too high near the peak cross section. Nevertheless, the Livermore group used 2-1-1 as the initial exciton numbers for the remaining calculations of the deuteron induced reactions for the CRP. 


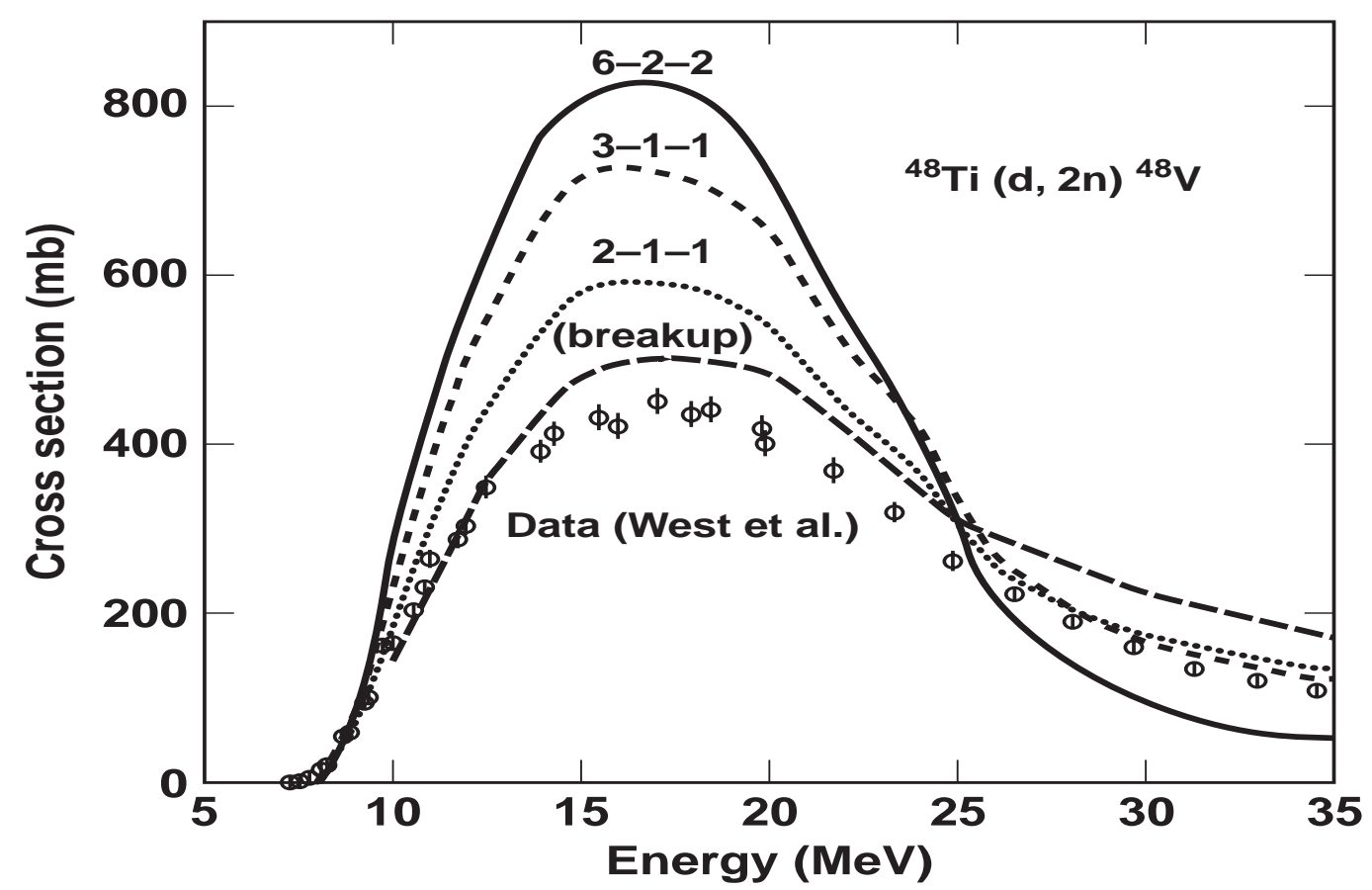

Fig. $1{ }^{48} \mathrm{Ti}(\mathrm{d}, 2 \mathrm{n})^{48} \mathrm{~V}$.

The cross sections including the deuteron breakup are compared with Alice-91 calculations with several choices of the initial exciton numbers (6-2-2, 3-1-1, 2-1-1) and the data of West et al. (1993). The numbers, e.g., 6-2-2, etc., refer to the initial exciton numbers in the hybrid preequilibrium model, 6 is the total exciton number (particles plus holes), 2 and 2 are the number of neutron and proton excitons above the Fermi energy.

Another important feature in modeling cross sections is the nuclear structures or structure effects that are included in the level density calculations. We show in Fig. 2 the cross sections for ${ }^{\text {nat }} \mathrm{Fe}(\mathrm{d}, \mathrm{x}){ }^{56} \mathrm{Co}$ reaction, where we compare the cross sections calculated with level densities from the Fermi gas (FG) model and those from the Kataria and Ramamurthy (KR) formalism which includes shell and pairing effects. These are the two level density options that are available in the Alice-91 code. The data in the figure is from Takacs et al. (1997) and Clark et al. (1969). We conclude from the results in Fig. 2 that the calculated cross sections may differ by a factor of two depending on the level density options used. We showed here one of the extreme cases for the reactions in this CRP. (The comparison of the modeled cross sections with the experimental data for other reactions is given in the next chapter.) It is worth to note that the calculations by the Obninsk group and those by Gul of Islamabad include shell effects but in a different way. Obninsk group also includes the collective effects in their level density calculations. 


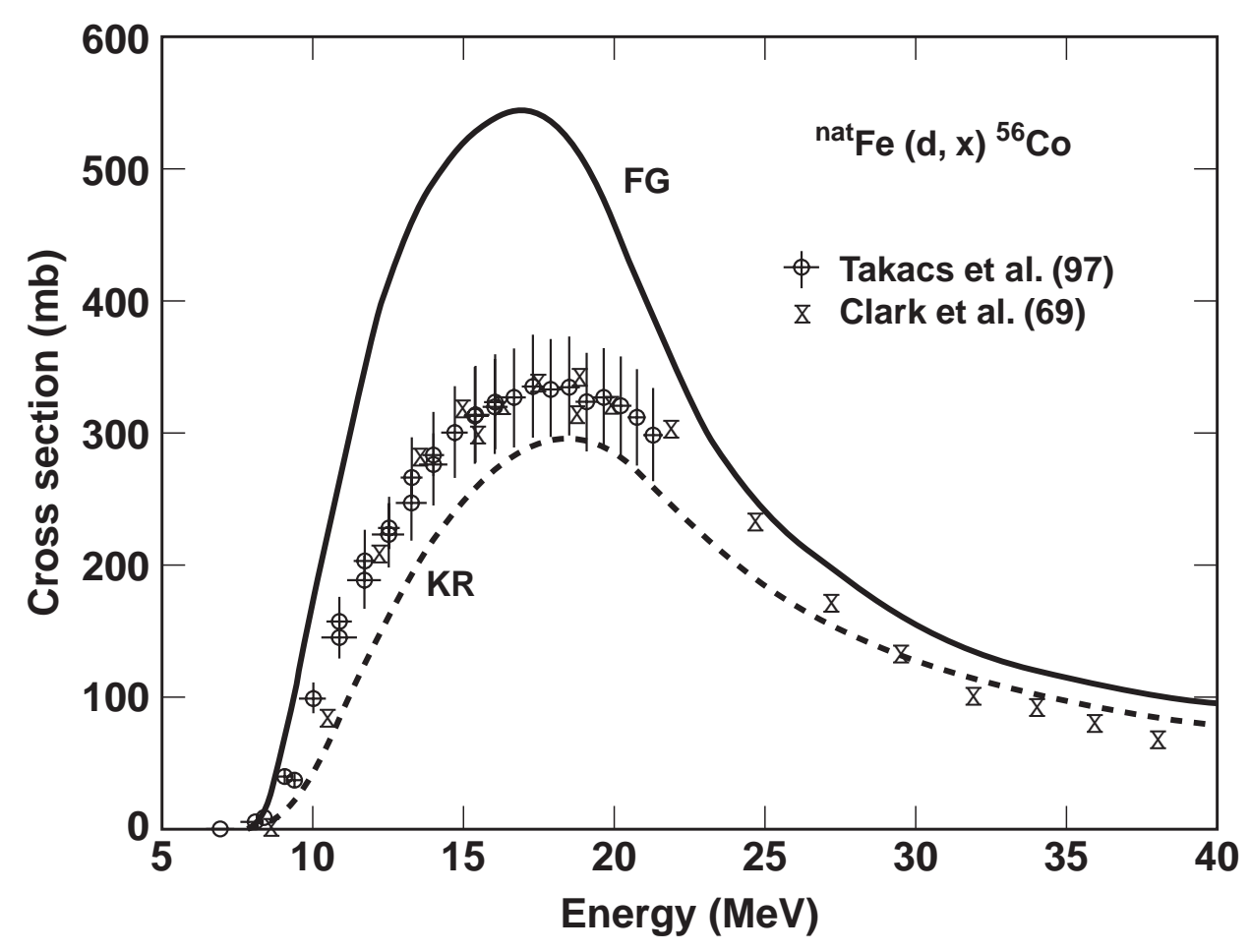

Fig. $2{ }^{\text {nat }} \mathrm{Fe}(\mathrm{d}, \mathrm{x})^{56} \mathrm{Co}$.

The FG stands for the Fermi gas model level densities and KR for the Kataria and Ramamurthy (1980) level densities with shell effects. The calculations were done using the Alice-91 code using the hybrid preequilibrium model and the Weisskopf-Ewing evaporation. The optical potentials used are described in Blann and Vonach (1983).

Fig. 3 A light target reaction showing poor fit by the preequilibrium-evaporation approach. (If needed more than one figure)

Fig. 4 Will show a good fit. For example an Alice -IPPE calculation

Fig 5. A figure from Spec calculations

Fig. 6. A figure from the Gul calculations

Note 1: I do not have all the figures to decide which figures to include in Figs. 3-6. I am waiting to see all the figures from Ferenc Tarkanyi

Note 2: I will add a paragraph discussing the overall accuracy of the calculations 


\section{References}

Akkermans, J. M. and Gruppelaar, H. (1985) Analysis of continuum gamma-ray emission in precompound-decay reactions. Phys. Lett. 157B, 95-100.

Aslam Lone, M. (1978) Title (?) . Proc. Third Int. Symp. on neutron capture gamma-ray spectroscopy and related topics, Editors: R. E. Chrien and W. R. Kane, Plennum Press, New York, p. 161.

Avrigeanu, A., Hodgson, P. E. and Avrigeanu, M. (1994) Global optical potentials for emitted alpha particles. Phys. Rev. C 49, 2136-2141.

Barnett, A. R., Feng, D. H., Steed, J. W. and Goldfarb, L. J. B. (1974), Computer Phys. Commun. 8, 377-395.

Becchetti, Jr., F. D. and Greenless, G. W. (1969) Nucleon-nucleus optical model parameters, A > 40, E < $50 \mathrm{MeV}$. Phys. Rev. 182, 1190-1209.

Bertini, H. W. (1963) Low-energy intranuclear cascade calculation. Phys. Rev. 131, 1801-1821.

Bisplinghoff, J. (1986) Configuration mixing in preequilibrium reactions: A new look at the hybrid-exciton controversy. Phys. Rev. C 33, 1569-1580.

Blann M. (1968) Extensions of Griffin's statistical model for medium-energy nuclear reactions. Phys. Rev. Lett. 21, 1357-1360.

Blann M. (1973) A-priori preequilibrium decay models. Nucl. Phys. A213, 570-588.

Blann, M. (1975) Preequilibrium decay. Annu. Rev. Nucl. Sci. 25, 123-166.

Blann, M. and Vonach, H. K. (1983) Global test of modified precompound decy models. Phys. Rev. C 28, 1475-1492.

Blann, M., Reffo, G. and Fabbri, F. (1988) Calculation of $\gamma$-ray cascades in code Alice. Nucl. Inst. Methods in Physics Research A265, 490-494.

Blann, M. (1991) Recent progress and current status of preequilibrium reaction theories and computer code ALICE. Lawrence Livermore National Laboratory Report, UCRL-JC-109052 (unpublished).

Blann, M., Gruppelaar, H., Nagel, P. and Rodens, J. (1994) International code and model intercomparison for intermediate energy nuclear data. NEA Data Bank, OECD, Paris.

Blann, M. (1996) New precompound decay model. Phys. Rev. C 54, 1341-1349.

Blokhin, A. I., Ignatyuk, A. V. and Shubin, Yu. N. (1988) Vibrational enhancement of the level density of nuclei in the iron region, Sov. J. Nucl. Phys. 48(2), 232-236.

Bohr, A. and Mottelson, B. (1974) Nuclear Structure, vol. 2, Benjamin Inc., New York and Amsterdam.

Chen, K., Fraenkel, Z., Friedlander, G., Grover, J. R., Miller, J. M. and Shimamoto, Y. (1968) VEGAS: A Monte Carlo simulation of intranuclear cascades. Phys. Rev. 166, 949-967.

Clark, J. W., Fulmer, C. B. and Williams, I. R. (1969) Excitation functions for radioactive nuclides produced by deuteron-induced reactions in iron. Phys. Rev. 179, 11041108.

Cugnon, J. , Volant, C. and Vuillier, S. (1997) Improved intranuclear cascade model for nucleon-nucleus interactions. Nucl. Phys. A620, 475-509.

Dilg, W., Schantl, W., Vonach, H. and Uhl, M. (1973) Level density parameters for the back-shifted Fermi gas model in the mass range $40<$ A $<250$. Nucl. Phys. A217, 269-298.

Dityuk, I., Konobeyev, A. Yu., Lunev, V. P. and Shubin, Yu. N. (1998) New version of the advanced computer code ALICE-IPPE, INDC (CCP)-410, International Atomic Energy Agency (IAEA), Vienna. 
Ericson, T. (1960) The statistical model and nuclear level densities. Adv. Phys. 9, 423511.

Ferrero, A., Gadioli Erba, E., Iori, I., Molho, N. and Zetta, L. (1979) $\alpha$-emission in proton induced reaction. Z. Phys. A293, 123-134.

Feshbach, H., Kerman, A. and Koonin, S. (1980) The statistical theory of multi-step compound and direct reactions. Ann. Phys. (NY) 125, 429-476.

Gadioli, E. and Hodgson, P. E. (1992) Pre-equilibrium nuclear reactions, Clarendon Press, Oxford.

Gadioli, E., Gadioli Erba, E. and Hoggan, J. J. (1977) Preequilibrium decay of nuclei with A 90 at excitation energies to $100 \mathrm{MeV}$. Phys. Rev. C 16, 1404-1424.

Gilbert, A. and Cameron, A. G. W. (1965) A composite nuclear-level density formula with shell corrections. Can. J. Phys. 43, 1446-1496.

Griffin, J. J. (1966) Statistical model of intermidate structure. Phys. Rev. Lett. 17, 478481.

Gudima, K. K., Mashnik, S. G. and Toneev, V. D. (1983) Cascade-exciton model of nuclear reactions. Nucl. Phys. A401, 329-360.

Gul, K. (1995) A computer code based on Hauser-Feshbach and Moldauer theory for nuclear cross section calculations, International Atomic Energy Agency (IAEA) Report INDC (PAK)-011, Vienna.

Gul, K. (1996) Theoretical formulation of PREMOD code based on geometry-dependent hybrid model of nuclear pre-equilibrium reactions, Scientific Information Division Report SID-7, PINSTECH, Islamabad.

Handbook for calculations of nuclear reaction data: Referenece input parameter library (1998), International Atomic Energy Agency (IAEA),Vienna, IAEA-TECDOC1034.

Hansen, G. and Jensen, A. (1983) Energy dependence of the rotational enhancement factor in the level density. Nucl. Phys. A406, 236-256.

Hauser, W. and Feshbach, H. (1952) The inelastic scattering of neutrons. Phys. Rev. 87, 366-373.

Hofstadter, R. (1957) Nuclear and nucleon scattering of high-energy electrons. Аnпи. Rev. Nucl. Sci. 7, 231-316.

Ignatyuk, A. V. (1983) Statistical Properties of Excited Atomic Nuclei (in Russian), Energoatomizdat, Moscow; Translated in English by the International Atomic Energy Agency (IAEA), IAEA Report INDC-233(L), Vienna, 1985.

Ignatyuk, A. V., Istekov, K. K. and Smirenkin, G. N. (1979) The role of collective effects in the systematics of nuclear level densities. Sov. J. Nucl. Phys. 29(4), 450-454.

Ignatyuk, A. V., Weil, J. L., Raman, S. and Kahane, S. (1993) Density of discrete levels in ${ }^{116}$ Sn. Phys. Rev. C 47, 1504-1513.

Iwamoto, A. and Harada, K. (1982) Mechanism of cluster emission in nucleon-induced preequilibrium reactions. Phys. Rev. C 26, 1821-1834.

Kataria, S. K. and Ramamurthy, V. S. (1980) Macrospic systematics of nuclear level densities. Nucl. Phys. A349, 10-28.

Kikuchi, K. and Kawai, M. (1968) Nuclear Matter and Nuclear Reactions, North Holland, Amsterdam.

Lane, A. M. and Thomas, R. G. (1958) R-matrix theory of nuclear reactions. Rev. Mod. Phys. 30, 257-353.

Michel, R. and Nagel, P. (1997) Title (?) . NSC/DOC(97)-1, NEA Data Bank, OECD, Paris.

Milazzo-Colli, L. and Braga-Marcazzan, G. M. (1973) $\alpha$-emission by preequilibrium 
processes in $(\mathrm{n}, \alpha)$ reactions. Nucl. Phys. A210, 297-306.

Mustafa, M. G. (1997) Projectile breakup and Hauser-Feshbach modeling. Proc. Int. Conf. on Nuclear Data for Science and Technology, Trieste, Italy, Editors: G. Reffo, A. Ventura and C. Grandi, pp. 274-276.

Myers, W. D. (1977) Droplet Model of Atomic Nuclei, IFI/Plenum, New York.

Myers, W. D. and Swiatecki, W. J. (1967) Anomalies in nuclear masses. Ark. Fys. 36, 343-352.

Nishioka, H., Weidenmuller, H. A. and Yoshida, S. (1988) Statistical theory of prcompound reactions: The multistep direct process. Ann. Phys. (NY) 183, 166187.

Oblozinsky, P., Ribansky, I. and Betak, E. (1974) Intermediate-state transition rates in the exciton model. Nucl. Phys. A226, 347-364.

Oblozinsky, P. and Ribansky, I. (1978) Emission rate of preformed $\alpha$-particles in preequilibrium decay. Phys. Lett. B74, 6-12.

Pearlstein, S. (1988) Nuclear Data for Science and Technology, Mito, Japan, p1 15.

Perey, F. G. (1963) Optical model analysis of proton elastic scattering in the range of 9 to $22 \mathrm{MeV}$. Phys. Rev. 131, 745-763.

Sato, N., Iwamoto, A. and Harada, K. (1983) Preequilibrium emission of light composite particles in the framework of the exciton model. Phys. Rev. C 28, 1527-1537.

Shen, Q. and Zhang, J. (1993) The SPEC Code. Commun. Nucl. Data Prog. 10, 53.

Takacs, S., Tarkanyi, F., Sonck, M., Hermanne, A. and Sudar, S. (1997) Study of deuteron induced reactions on natural iron and copper and their use for monitoring beam parameters and for thin layer activation technique. Proc. $14^{\text {th }}$ Int. Conf. on Applications of Accelerators in Research and Industry, Denton, Texas, November 1996. Editors: J. L. Dugan and I. L. Morgan, AIP Conference Proceeding 392, AIP, Woodbury, New York, p. 659.

Tamura, T., Udagawa, T. and Lenske, H. (1982) Multistep direct reaction analysis of continuum spectra in reactions induced by light ions. Phys. Rev. C 26, 379-404.

Udagawa, T. and Tamura, T. (1981) Derivation of breakup-fusion cross sections from the optical theorem. Phys. Rev. C 24, 1348-1349.

Udagawa, T. and Tamura, T. (1986) Formulation of elastic and inelastic breakup-fusion reactions. Phys. Rev. C 33, 494-503.

Vernotte, J., Berrier-Ronsin, G., Kalifa, J. and Tamisier, R. (1982) Optical model analysis of ${ }^{3} \mathrm{He}$ elastic scattering from s-d shell nuclei at $25 \mathrm{MeV}$. Nucl. Phys. A390, 285313.

Weisskopf, V. F. and Ewing, D. H. (1940) On the yield of nuclear reactions with heavy elements. Phys. Rev. 57, 472-485.

West, Jr., H. I., Lanier, R. G., Mustafa, M. G., Nuckolls, R. M., Nagle, R. J., O’Brien, H., Frehaut, J., Adam, A and Philis, C. (1993) Some light-ion excitation-function measurements on titanium, yttrium, and europium, and assosciated results. Lawrence Livermore National Laboratory Report UCRL-ID-115738 (unpublished).

Wilkinson, D. H. (1960) Nuclear Spectroscopy (Part B) Editor: Fay Azjenberg-Selov, Academic Press, New York, p. 644.

Williams, Jr., F. C. (1971) Particle-hole state density in the uniform spacing model. Nucl. Phys. A166, 231-240.

Wilmore, D. and Hodgson, P. E. (1974) The calculation of neutron cross-sections from optical potentials. Nucl. Phys. 55, 673-694.

Zhang, J., Wen, Y., Wang, S. and Shi, X. (1988) Formation and emission of light 
particles in fast neutron induced reactions-a unified compound pre-equilibrium model. Commun. Theor. Phys. (Beijing, China) 10, 33-44.

Zhang J., Yan, S. and Wang, C. (1992) The pick-up mechanism in composite particle emission processes. Z. Phys. A344, 251-258. 